\title{
The influence of corticosteroids and Azelastine on epithelial cell integrity in chronic rhinosinusitis with polyps*
}

\author{
Nicolas Vogel', Seraina Kunz', Cezmi Akdis², Michael B. Soyka \\ ' University Hospital of Zurich, Department of Otorhinolaryngology, Frauenklinikstrasse 24, 8091 Zurich, Switzerland \\ ${ }^{2}$ Swiss Institute of Allergy and Asthma research, Hermann-Burchard-Strasse 9, 7265 Davos Wolfgang, Switzerland
}

Rhinology Online, Vol 3: 8 - 14, 2020

http://doi.org/10.4193/RHINOL/20.004

*Received for publication:

January 15,2020

Accepted: March 13, 2020

Published: March 18, 2020

\begin{abstract}
Background: The pathogenesis of chronic rhinosinusitis with nasal polyps (CRSwNP) is still not completely understood. Regarding the pathogenesis an impaired barrier function of the sino-nasal epithelium has been shown in CRS. Intranasal corticosteroids are widely used for treatment and part of current guidelines. This study aims to analyse the influence of corticosteroids on the epithelial integrity in air-liquid-interface (ALI) epithelial cell cultures of patients with CRSwNP.

Methodology: Polyp tissue from 11 patients with CRSwNP undergoing a sphenoethmoidectomy was collected. The epithelial cells were cultured in ALI. After differentiation of the cells, the permeability of the cell layer was defined by measuring the transepithelial resistance (TER). Commonly used corticosteroids, (Fluticasone \& Mometasone) and Azelastine were added and their impact on the TER was documented and compared to the TER of ALI cultures without additives (controls).

Results: After 48 hours, Fluticasone showed a significant increase of the TER. Mometasone and Azelastine had no significant impact on the TER. The paracellular passage of Dextran molecules in ALI cultures showed a negative correlation to the TER. Preoperative in vivo corticosteroid treatment had no effect on the baseline TER. Further analysis of the impact of preoperative corticosteroid treatment showed no effect on polyp size, sense of smell, histopathologic eosinophilic count and allergic sensitization.
\end{abstract}

Conclusion: Fluticasone shows a direct effect in restoring sino-nasal epithelial barrier in-vitro, even in the absence of inflammatory cells.

Key words: paranasal sinus disease, nasal polyps, sinusitis, nasal mucosa, nose

\section{Introduction}

Chronic rhinosinusitis with nasal polyps (CRSwNP) is a highly prevalent ${ }^{(1)}$ condition with remarkable impact on quality of life $(2,3)$. Furthermore, there is a massive burden of costs regarding the necessary consultations and medical treatment ${ }^{(4-6)}$ of the patients as well as absenteeism associated with the disease. Recently the Cochrane review of $2016^{(7,8)}$ came to the same conclusion as already described in the EPOS guidelines ${ }^{\left({ }^{9}\right)}$ - basic treatment should include nasal irrigation and the use of intranasal corticosteroids.

Although the treatment guidelines are based on clear evidence, the pathogenesis - in particularly the involved factors contributing to it - are just partly understood. We have previously shown loss of epithelial integrity in sino-nasal epithelial cells in CRS leading to the theory of the impaired epithelial barrier ${ }^{(10)}$. It has been hypothesized that there may be genetic background or an environmental or infectious triggering event leading to a continuous disturbance and barrier dysfunction. A disrupted epithelial barrier may allow the penetration of allergens, toxic substances or bacteria by a paracellular route. In 2010, Sachse et al. provided evidence for penetrating Staphylococcus aureus towards the submucosal layer in patients with CRSwNP (11). 
It has been shown, that in allergic airway diseases (e.g. allergic rhinitis; AR) there is a disturbance of the epithelial barrier function as well ${ }^{(12,13)}$. In these patients, glucocorticoids seem to influence the barrier function in a positive way ${ }^{(14)}$. It is well known so far that glucocorticoids reduce inflammatory processes at different sites ${ }^{(15)}$. Looking specifically at the in-vitro effect of Fluticasone propionate (FP) on epithelial cell ALI cultures in $A R$, it has been shown that there is an improvement in barrier function ${ }^{(13)}$.

As mentioned above, in CRSwNP we are aware of a decreased barrier function and we have evidence for the treatment with intranasal corticosteroids: this evidence is based on symptom scores, polyp size and other parameters, but there was no investigation of the effect of commonly used corticosteroids on the epithelial barrier function of cells from CRSwNP patients.

Hence, we aimed to investigate this influence of common topical steroids on human sino-nasal epithelial cells in order to provide evidence for an additional way of action. Furthermore, there are available topical combinations of corticosteroids with antihistamines for which reason Azelastine was selected additionally to investigate its sole potential on the epithelial barrier.

\section{Materials and Methods}

\section{Polyp tissue}

Polyp tissue of patients with CRSwNP was obtained in patients undergoing a standard endoscopic sinus surgery. Intraoperatively, none of the patients received intranasal or intravenous corticosteroids or antihistamines. Half of the tissue was sent to histopathological analyses while the other half was used for cell cultures. All of the patients signed an informed consent for participating in this study. Ethical permission was given by the ethical committee of the Kanton of Zurich (KEK 2011-0514).

\section{Cell cultures of human sino-nasal epithelial cells}

Polyps were cut in pieces and primary human sino-nasal epithelial cells were isolated from the tissue using trypsine (TrypsineEDTA 0.5\%,15400054, Gibco, Thermo Fisher Scientific, Waltham, USA). Trypsine was then neutralized with Trypsin neutralizing solution (TNS, CC-5002, Lonza, Walkersville, USA) and the tissue

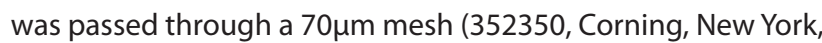
USA). The obtained suspension was centrifuged, and the cell pellet was then resuspended in Bronchial epithelial cell growth medium (BEGM, CC-3171, Lonza). The suspension was seeded to standard T-75-Flasks (90075, TPP, Trasadingen, $\mathrm{CH}$ ) for further culture. Nutrition of the cells was always provided by Bronchial epithelial growth medium. Growth factors, cytokines and supplements were added individually (CC-4175, Lonza) without adding hydrocortisone to the medium. After growing the cells to approximately $80 \%$ confluence, they were detached by using Trypsin. We used TNS again to neutralize Trypsin, centrifuged the cells and resuspended the cell pellet with growth medium:
The medium was composed of a 1:1 mixed solution of Dulbeccos modified Eagle's medium (DMEM, BE12-741F, Lonza) and BEGM. Hydrocortisone and Triiodthyronine were excluded from the supplements. All-trans-retinoid acid solution (15ng/ $\mathrm{ml}$; 50nM) was added individually (R2625-50MG, Sigma-Aldrich, Missouri, USA). The cells were subsequent transferred to an Air-Liquid-interface (ALI) on transwell inserts (CLS3495-24EA, Corning, New York, USA). Medium was added in the apical and basal chamber of the ALI. ALI were checked daily microscopically and by measuring TER until differentiation. Growth medium was changed every second day in the apical and basal chamber. As soon as the cells reached confluence, the apical medium was removed. The basal chamber was changed in the same rhythm. Differentiation was reached if TER exceeded $200 \Omega{ }^{*} \mathrm{~cm}^{2}$. After differentiation either $0.1 \mu \mathrm{mol} / \mathrm{L}$ Fluticasone propionate (FP, PHR1702-200MG, Sigma-Aldrich), 0.176 umol/L Mometasone furoate (MF, PHR1400-500MG, Sigma-Aldrich) or $0.61 \mu \mathrm{mol} / \mathrm{L}$ Azelastine hydrochloride (AZE, PHR1636-1G, Sigma-Aldrich) were added in the medium of the basal chamber. The concentrations were calculated from commonly used topical steroids and from existing literature. Dimethyl sulfoxide (DMSO) was used for solubility of the substance and was therefore used without additives as the control group. In the control group DMSO (D2650, Sigma-Aldrich) was used in a concentration of $420 \mu \mathrm{mol} / \mathrm{L}$. TER was measured in triplicates after $24 \mathrm{~h}, 48 \mathrm{~h}, 72 \mathrm{~h}$ and $96 \mathrm{~h}$. For the measurement of TER, we used a Volt-Ohm-Meter (VOM, EVOM2, World Precision Instruments, Sarasta, USA).

Paracellular flux was determined by using FITC-Dextran (FITCDextran, FD4-100MG, Sigma-Aldrich). We used a final concentration of FITC-Dextran in ALI growth medium of $2 \mathrm{mg} / \mathrm{ml}$. The growth medium with FITC-Dextran was added in the apical chambers of several ALI. For every sample $200 \mu$ l were transferred on a 96-well plate suitable for analysis in an ELISA-Reader. The first sample was taken after 2 hours and then after day 1,2, 3,4 and 5. At each time the TER was measured before taking the samples. Samples were taken from the apical as well as from the basal chamber. An ELISA-Reader was used at $485.528 \mathrm{~nm}$ wavelength to measure the amount of FITC-Dextran in the samples.

\section{Statistical analysis}

All statistical calculations were done by using GraphPad Prism (Version 7.03). Mean \pm standard deviation was computed. We used the Mann-Whitney-U-Test for calculation of the results of the TER. Correlation of Dextran flux was calculated by using the Spearman-Coefficient.

It is important to mention that the results, as shown in the figure, are composed in the following way: if there were more than one ALI per experiment point of a patient, we calculated the mean TER for the calculation. 
Table 1. Characteristics of the included patients.

\begin{tabular}{lr}
\hline \multicolumn{1}{c}{ Total Patients } & \multicolumn{1}{c}{$\mathbf{1 1}(\mathbf{1 0 0} \%)$} \\
\hline Sex & \\
Women & $5(45 \%)$ \\
Men & $6(55 \%)$ \\
\hline Age & 45.6 years $( \pm 13.4)$ \\
Women & 49.4 years $( \pm 15.9)$ \\
Men & 42.5 years $( \pm 11.4)$ \\
\hline Preoperative corticosteroid treatment & $7(64 \%)$ \\
Systemically & $2(18 \%)$ \\
Topical & $5(45 \%)$ \\
Polyp size & \\
Grade I & $2(18 \%)$ \\
Grade II & $5(45 \%)$ \\
Grade III & $1(9 \%)$ \\
Grade IV & $3(27 \%)$ \\
Allergic sensitization & \\
Yes & $4(36 \%)$ \\
No & $5(45 \%)$ \\
N/a & $2(18 \%)$ \\
\hline Smokers & $2(18 \%)$ \\
\hline
\end{tabular}

\section{Results}

Subjects and patient characteristics

We recruited patients with CRSwNP undergoing a planned sphenoethmoidectomy. After written informed consent, we included 11 patients in the study. We analysed ALI cell cultures of all 11 patients. The patient characteristics are shown in Table 1.

\section{Analysis of the ALI cell cultures}

There were $53 \mathrm{ALI}$, which showed cellular differentiation. FP, MF and AZE was used for treatment of the ALI cultures und further compared to the control group (DMSO).

ALIs were divided according to the following protocol: DMSO as control group was used in $10 \mathrm{ALIS}$, FP was used in $16 \mathrm{ALIS}, \mathrm{MF}$ was used in $15 \mathrm{ALIs}$ and AZE was used in $12 \mathrm{ALIs}$. The measured TER was always compared to the initial value. Figure 1 shows the relative TER change compared to the baseline TER, which represents $100 \%$.

FP showed a statistically significant increase of the TER after $48 \mathrm{~h}$ compared to the control group $(131.1 \% \pm 53.4 \%, p=0.04)$. Looking at MF we found an improving of the TER after $48 \mathrm{~h}$ although without reaching statistical significance. The results are shown in Figure 1. AZE had no impact on the TER. Figure 2 is showing the means of the TER of one representative patient as an example. An overview of all results per substance is provided in Table 2 . In addition, we investigated the paracellular passing of molecules by using FITC-Dextran and related these results to the TER. The paracellular flux of FITC-Dextran molecules was measured daily over 5 days. After measurements of fluorescence at $485.528 \mathrm{~nm}$ ALI with not reproduceable intensity of absorption were excluded. Hence, for the measurements $8 \mathrm{ALI}$ cultures were

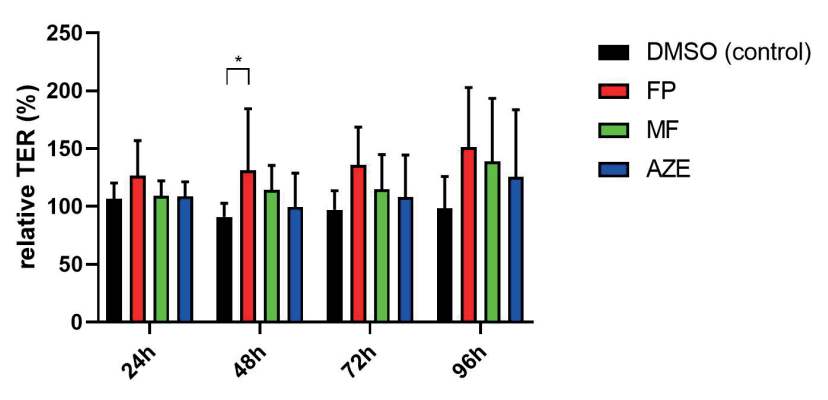

Figure 1. Each bar represents the effect of the added substance as the relative change in TER (\%) compare to T0 (baseline before addition of component). Whiskers show standard deviation. FP shows a significant increase of the TER after $48 \mathrm{~h}(\mathrm{p}=0.04) . \mathrm{n}=53 \mathrm{ALIs}$.

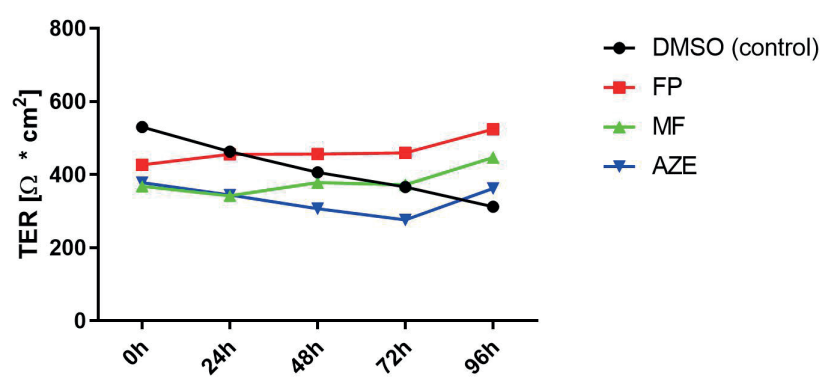

Figure 2. Shows the TER $\left[\Omega{ }^{*} \mathrm{~cm} 2\right]$ at given time points before (T0) and after the addition of the substance for one representative patient. $n=7$ ALIs.

included. The permeability for Dextran molecules was negatively correlated with the TER $(r=-0.76 ; p=0.037)$. The results are shown in Figure 3.

Furthermore, we analysed the clinical and histopathological presentation in relation to the TER of the individuals. We used the baseline TER of the differentiated ALI cell cultures before adding the substances. In the comparison of preoperative patients, who were using corticosteroid treatments regarding epithelial integrity, the group with systemic treatment showed a trend toward higher TER ( $p=0.19)$. The preoperative sense of smell had no effect on the TER ( $p=0.29)$. At the day of hospital admission, the polyp size of the patients had been documented. There were no significant differences of the TER between the polyp sizes. The eosinophil count of the histopathological result of the tissue had been either described as slight or pronounced eosinophilia. There were no differences between the different histologic groups $(p=0.18)$. Looking at the allergic sensitization, specifically on the IgE level, there was no difference of the TER between the group with allergic sensitization and the group without $(p=0.56)$.

\section{Discussion}

Our results showed for the first time a statistically significant 
Table 2. Numeric results of the TER regarding the subgroups.

\begin{tabular}{|c|c|c|c|c|}
\hline Group & mean ( \pm SD) & difference $( \pm S D)$ & difference \% ( \pm SD) & p-value \\
\hline \multicolumn{5}{|l|}{ DMSO (control) } \\
\hline $\mathrm{Oh}$ & $437.5(217.6)$ & - & - & - \\
\hline $24 \mathrm{~h}$ & $476.3(273.4)$ & $49.4(67.4)$ & $6.5(13.8)$ & - \\
\hline $48 \mathrm{~h}$ & $381.4(165.2)$ & $-28.6(69.3)$ & $-9.1(11.8)$ & - \\
\hline $72 \mathrm{~h}$ & $427.8(244)$ & $33.4(50.3)$ & $-2.8(16.5)$ & - \\
\hline $96 \mathrm{~h}$ & $460.9(265.7)$ & $63.9(73.9)$ & $-1.6(27.6)$ & - \\
\hline \multicolumn{5}{|c|}{ mometasone furoate } \\
\hline $\mathrm{Oh}$ & $409.4(184.4)$ & - & - & \\
\hline $24 \mathrm{~h}$ & $468.8(249.3)$ & $45.2(72)$ & $9.3(12.9)$ & 0.955 \\
\hline $48 \mathrm{~h}$ & $445.7(160.7)$ & $36.1(67.3)$ & $14.3(21.2)$ & 0.088 \\
\hline $72 \mathrm{~h}$ & $445.7(168.1)$ & 36.1 (73.9) & $14.8(30.1)$ & $>0.999$ \\
\hline $96 \mathrm{~h}$ & $517(169.5)$ & $104.5(121.1)$ & $38.9(54.5)$ & 0.622 \\
\hline \multicolumn{5}{|c|}{ fluticasone propionate } \\
\hline $\mathrm{Oh}$ & $392(208.1)$ & - & - & \\
\hline $24 \mathrm{~h}$ & $525.9(283.9)$ & $107.5(105.3)$ & $26.5(30.6)$ & 0.234 \\
\hline $48 \mathrm{~h}$ & $458.7(151)$ & $66.5(121.1)$ & $31.1(53.4)$ & 0.043 \\
\hline $72 \mathrm{~h}$ & $491.7(181.6)$ & $99.8(95.2)$ & $35.7(32.9)$ & 0.142 \\
\hline $96 \mathrm{~h}$ & $552.5(184.4)$ & $145(121.7)$ & $51.3(51.5)$ & 0.202 \\
\hline \multicolumn{5}{|c|}{ azelastine hydrochloride } \\
\hline $\mathrm{Oh}$ & $400.2(231.7)$ & - & - & \\
\hline $24 \mathrm{~h}$ & $463.5(252.9)$ & $32(41.4)$ & $8.9(12.6)$ & $>0.999$ \\
\hline $48 \mathrm{~h}$ & $366.2(158.4)$ & $-34(114)$ & $-0.5(29.4)$ & 0.945 \\
\hline $72 \mathrm{~h}$ & $408.4(215.9)$ & $8.3(111.8)$ & $8.3(36.2)$ & 0.761 \\
\hline $96 \mathrm{~h}$ & $490.4(240.3)$ & $65.3(134.9)$ & $25.6(58.2)$ & 0.792 \\
\hline
\end{tabular}

Results of the TER and differences in relation to the measurement at $0 \mathrm{~h}$ (T0). All absolute values are mentioned in $[\Omega * \mathrm{~cm} 2]$. P-values are calculated by using the differences of the TER of a substance related to the difference of the control group at given point in time.

gain of TER - representing the integrity of the epithelial barrier - after in vitro treating primary human sino-nasal epithelial cells of patients with CRSwNP with FP, a commonly used corticosteroid. This shows a substantial impact of FP on the barrier function of human sino-nasal epithelial cells. MF showed an overall trend of improvement, while AZE had no significant impact on the TER.

CRSwNP is a highly prevalent disease ${ }^{(1)}$ with widely accepted treatment guidelines using intranasal corticosteroids ${ }^{(7,9)}$. Despite these guidelines there is still no scientific evidence looking on the impact of the corticosteroids on the sino-nasal epithelia especially relating to the integrity of the epithelium. In the present study, we investigated how commonly used intranasal corticosteroids influence the permeability - measured by TER - of in vitro sino-nasal epithelial cells.

Assuming that a disturbance of the epithelial barrier is one contributing factor underlying the disease of CRSwNP ${ }^{(10)}$, this study is showing a restoration of the integrity of the epithelium by corticosteroids even in the absence of inflammatory cells. Oral corticosteroid treatment in CRSwNP leads to a significant reduction of inflammatory cytokines such as Interferon- $\gamma$ (IFN- $\gamma$ ), Interleukin-4 (IL-4) and IL-5 in neutrophilic as well as in eosinophilic inflammation. But there was no effect on IL-8, IL-17 and the neutrophilic count. In patients with neutrophilic inflammation there was no reduction of clinical symptoms after treatment. Hence, neutrophilic count and neutrophilic inflammatory cytokines can be used as predictor for corticosteroid efficacy ${ }^{(16)}$. Proinflammatory cytokines, such as IL-5, IL-6, IL-8, granulocytemacrophage colony-stimulating factor (GM-CSF) and others are relevant for inducing chemotaxis, activity and survival of eosinophils in the sino-nasal mucosa. Treating CRS patients with prednisolone showed a significant reduction of IL-5, IL-8 and GM-CSF in in vitro analysis of fresh tissue of human sino-nasal epithelial cells. Unfortunately, there is lack of information about which CRS entity had been analysed ${ }^{(17)}$. In 2011 Bleier et al. challenged cell cultures of human sino-nasal epithelial cells with 


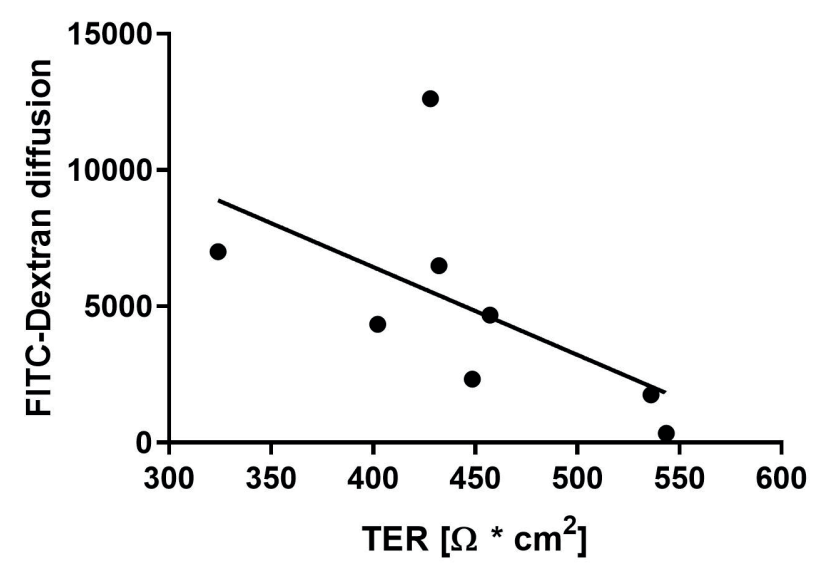

Figure 3. Negative correlation of TER and FD4 passage. The FD4 passage is expressed as the measured fluorescein intensity. For the measurement, we used 8 ALIs with reproduceable intensity measurements of fluorescence absorption. TER is mentioned in $\left[\Omega{ }^{*} \mathrm{~cm}^{2}\right]$.

lipopolysaccharides and showed that there is a significant decrease of IL- 6 and IL- 8 secretion in the presence of dexamethasone. Furthermore, they measured the dynamics of the TER of ALI cultures, which were exposed to cigarette smoke extract. There was a significant decrease after 90 minutes. Unfortunately, there were no TER measurements mentioned in relation to dexamethasone treatment ${ }^{(18)}$.

Our ALI cell culture system is fully free of inflammatory cells, as only sino-nasal epithelial cells were cultured in the ALI systems. Therefore, the observed effects of corticosteroids on epithelial integrity must be directly triggered by the drug interaction with epithelial cells without having an impact on inflammation and immune cells itself.

Summing up, we see at least a dual role of corticosteroids in the treatment of CRSwNP. First, the direct effect on closure of tight junctions by Fluticasone, second, the inhibition of inflammation and thereby reduction of proinflammatory cytokines including IFN- $\gamma$ and IL-4, which are drivers of barrier deficiency in CRS ${ }^{(10)}$. But the effects of steroids on epithelial cytokine release and their relevance or relation in TJ dysfunction in absence of inflammatory cells remains to be further elucidated. Especially a study protocol looking at all the above-mentioned cytokines, the TER, immunostaining and mRNA during a treatment with corticosteroids is still missing but would be important for further understanding of the relations of these parameters in CRSWNP. However, not all patients with CRSwNP are responders to the treatment of intranasal corticosteroids ${ }^{(19,20)}$. Differences in glucocorticoid receptors (hGRa and hGR 3 ) could explain some steroid resistance in asthma and potentially also in CRS. Such factors could have had an influence on our results as well, since we had not investigated, whether patients were clinical responders to steroids. Furthermore, selection bias with an accumulation of "non-responders" could also influence our results.

The impact of corticosteroids on the cells of the epithelial barrier itself has to be understood: In 2012 Sekiyama et al. investigated the influence of corticosteroids (Dexamethasone, Budesonide and Fluticasone propionate) on the epithelial barrier in bronchial epithelial cells and showed an enhanced expression of tight junction (TJ) proteins (zonula occludens-1 (ZO-1), occluding) directly at the site of the $\mathrm{TJ}^{\left({ }^{14}\right)}$. Furthermore, it has been shown that the nuclear erythroid 2-related factor 2 (Nrf2) in human bronchial epithelial cells is responsible for the effect of the corticosteroids leading to the enhanced expression of TJ proteins ${ }^{(21)}$. In summary these findings may explain the pathway how epithelial cells react with a gain of the TER on corticosteroids without involving inflammatory cells in human bronchial cells. To further proof this theory, Steelant et al. ${ }^{(13)}$ used the same setting of ALI cell cultures as we did, in investigating the influence of FP on cells of patients suffering from allergic rhinitis (AR). The results showed an altered occludin and ZO-1 expression with a decreased TER in patients with AR. After treating the ALI cell cultures with FP, they showed a prevention of the IL-4 induced barrier dysfunction: The TER was significantly increased in this group. Regarding the study protocol they used hydrocortisone in the medium for harvesting the cells. In our study we excluded the use of hydrocortisone explicitly to make sure that the effect of the added substances is not biased in any way before. In general, barrier function is dependent of more than the above-mentioned parameters: Recent research provided evidence for further explanation of barrier disruption: Human sino-nasal epithelial cells release IL-33 as a reaction to surrounding pollutants, allergens, etc. IL-33 is stimulating a Th2 driven inflammation which leads to further disruption of the barrier function ${ }^{(22)}$. Breaking down this information we must constitute that there is a self-sustentative process of barrier disruption through epithelial cells.

Looking at the inflammatory pathway there are other interesting parameters which could influence the integrity of the epithelial barrier: Beside IL-33 there are other "alarmins" such as IL-25 and thymic stromal lymphopoietin (TSLP). IL-25 is another providing factor for Th2 driven inflammation and was shown to be increased in polyp tissue - as well as IL-33 and TSLP (23,24). TLSP is an epithelial cell derived cytokine which is likely to have an impact on Th2 driven inflammation through dendritic cells as wells as T-cells ${ }^{(25)}$. As above mentioned, the Th2 driven inflammation is leading to a disruption of the barrier function and is promoted by IL-25, IL-33 and TSLP. Contrariwise there is evidence that the TER is significantly increased through TSLP (26). It appears that the important key in the inflammatory process and the corresponding barrier function is the individual regulation and proportion of these cytokines.

For further evaluation of the impact on the epithelial integrity a model analysing the correlation between TSLP production and 
the TER in relationship to production of IL-25 and IL-33 would be interesting and may could be used as target for influencing barrier function.

There is evidence that histone deacetylase (HDAC) activity is inversely correlating with barrier function in AR. This brings up the discussion of epigenetic alterations as parameters of barrier function. By blocking HDAC activity it was possible to restore barrier integrity on different levels: The TER, immunostaining of TJ proteins and measurement of mRNA levels of the proteins showed a significant increase. These findings are important to understand the multilevel and multiparametric pathogenesis of barrier dysfunction as well as the importance of the cell layer integrity for a healthy subject ${ }^{(27)}$. Interestingly, HDAC activity can be inhibited by sodium butyrate which is an end product of the gut microbiome ${ }^{(28)}$. Further research must be done to evaluate the therapeutic effect of sodium butyrate on the barrier function in CRS.

Current guidelines recommend the use of topical steroids mainly on the premise of lowering the inflammatory load. Our results suggest that the effects of the drug are beyond these mechanisms, potentially even preventing further chronification by increasing the barrier function. This fact could further encourage clinicians in the use of these drugs and improve patients' compliance by more detailed explanation of its way of action. In our results we could not see an effect of topical or systemic steroids use prior to surgery on the in-vitro TER measurements. This could be partly due to underpowering, however, it is much more likely that the effect is lost during passaging and culturing of cells in the absence of proinflammatory cytokines and cells. Therefore, the cells seem to return to its native impaired barrier function.

In intranasal application of topical corticosteroids or antihistamines the substance is placed apically on the epithelial cells. In the ALI model it is of outmost importance to keep the apical cells in touch with air: The contact to the air is one underlying stimulus for complete differentiation of the cells in the ALI model. Therefore, we chose to add the substances in the basolateral chamber to ensure full differentiation.

Application in vivo is punctually, and the substances are cleared by mucociliary clearance within minutes. In vitro the substances are in touch with the cells for a longer time. The difference between these two models is that in vivo there are inflammatory cells which can intensify or decrease the reaction of epithelial cells by cytokines. In our model there are no inflammatory cells. Thus, a longer contact of the substances is needed. The focus of this study lies in investigating the in vitro effect of the mentioned substances on the TER. The further analyses of the baseline TER regarding different parameters (preoperative corticosteroid treatment, preoperative sense of smell, polyp size, allergic sensitization) provides additional information. For the authors these results must be interpreted with caution due to underpowering what is a limitation to answer these questions. Although our investigation only included 11 subjects, we are confident to present relevant and consistent data. Future investigations in larger groups could prove our findings on a protein and mRNA level as well as direct effects of topical steroids in vivo, i.e. using trans tissue resistance measurement before and after treatment. The sample size of patients is a limitation of our study. Particularly, when looking at the effects of polyp size, allergic sensitization, sense of smell and eosinophil count. Nevertheless, more than $50 \mathrm{ALI}$ cultures could be established, which is an adequate count considering the time consuming and sophisticated technique.

A further strength of our study is the use of fresh sino-nasal epithelial cells. It is important to be aware that the results of experiments with sino-nasal epithelial cells are dependent on the decision which cells are used: Immortal cell lines (e.g. Calu-3) show different immune and epithelial barrier response than fresh sino-nasal epithelial tissue ${ }^{(29)}$.

\section{Conclusions}

In conclusion these results show for the first time an association between the use of corticosteroids and the restoration of the impaired epithelial barrier in chronic rhinosinusitis with polyps.

\section{Acknowledgement}

Not applicable.

\section{Authorship contribution}

NV wrote the manuscript. NV and SK conducted the work in the cell culture laboratory. CA revised the whole manuscript before submitting. MBS guided the laboratory work and the writing process of the manuscript. He revised the manuscript before submitting.

\section{Conflict of interest}

There is no conflict of interest.

\section{Ethics approval and consent to participate}

All of the patients signed an informed consent for participating in this study. Ethical permission was given by the ethical committee of the Kanton of Zurich (KEK 2011-0514).

\section{Consent for publication}

Not applicable.

\section{Availability of data and materials}

The datasets used and/or analysed during the current study are available from the corresponding author on reasonable request.

\section{Funding}

There was no funding. 


\section{References}

1. Hastan D, Fokkens WJ, Bachert C, Newson RB, Bislimovska J, Bockelbrink A, et al. Chronic rhinosinusitis in Europe--an underestimated disease. A GA2LEN study. Allergy. 2011;66(9):1216-23.

2. Alt JA, Smith TL. Chronic rhinosinusitis and sleep: a contemporary review. Int Forum Allergy Rhinol. 2013;3(11):941-9.

3. Erskine S, Hopkins C, Kumar N, Wilson J, Clark A, Robertson A, et al. A cross sectional analysis of a case-control study about quality of life in CRS in the UK; a comparison between CRS subtypes. Rhinology. 2016:54(4):311-5.

4. Bhattacharyya N. Incremental health care utilization and expenditures for chronic rhinosinusitis in the United States. Ann Otol Rhinol Laryngol. 2011;120(7):423-7.

5. Caulley L, Thavorn K, Rudmik L, Cameron C, Kilty SJ. Direct costs of adult chronic rhinosinusitis by using 4 methods of estimation: Results of the US Medical Expenditure Panel Survey. J Allergy Clin Immunol. 2015;136(6):1517-22.

6. Rudmik L. Economics of Chronic Rhinosinusitis. Curr Allergy Asthma Rep. 2017;17(4):20

7. Chong LY, Head K, Hopkins C, Philpott C, Schilder AG, Burton MJ. Intranasal steroids versus placebo or no intervention for chronic rhinosinusitis. Cochrane Database Syst Rev. 2016;4:CD011996.

8. Chong LY, Head K, Hopkins C, Philpott C, Glew S, Scadding G, et al. Saline irrigation for chronic rhinosinusitis. Cochrane Database Syst Rev. 2016;4:CD011995.

9. Fokkens WJ, Lund VJ, Mullol J, Bachert C, Alobid I, Baroody F, et al. European Position Paper on Rhinosinusitis and Nasal Polyps 2012. Rhinol Suppl. 2012(23):3 p preceding table of contents, 1-298.

10. Soyka MB, Wawrzyniak P, Eiwegger T, Holzmann D, Treis A, Wanke $K$, et al. Defective epithelial barrier in chronic rhinosinusitis: the regulation of tight junctions by IFN-gamma and IL-4. J Allergy Clin Immunol. 2012;130(5):1087-96.e10.

11. Sachse F, Becker K, von Eiff C, Metze D, Rudack C. Staphylococcus aureus invades the epithelium in nasal polyposis and induces IL-6 in nasal epithelial cells in vitro. Allergy. 2010;65(11):1430-7.
12. Georas SN, Rezaee F. Epithelial barrier function: at the front line of asthma immunology and allergic airway inflammation. J Allergy Clin Immunol. 2014;134(3):509-20.

13. Steelant B, Farré R, Wawrzyniak P, Belmans J, Dekimpe E, Vanheel $H$, et al. Impaired barrier function in patients with house dust mite-induced allergic rhinitis is accompanied by decreased occludin and zonula occludens-1 expression. J Allergy Clin Immunol. 2016;137(4):1043-53.e1-5.

14. Sekiyama A, Gon Y, Terakado M, Takeshita I, Kozu Y, Maruoka S, et al. Glucocorticoids enhance airway epithelial barrier integrity. Int Immunopharmacol. 2012;12(2):350-7.

15. Mullol J, Obando A, Pujols L, Alobid I. Corticosteroid treatment in chronic rhinosinusitis: the possibilities and the limits. Immunol Allergy Clin North Am. 2009;29(4):657-68

16. Wen W, Liu W, Zhang L, Bai J, Fan Y, Xia W, et al. Increased neutrophilia in nasal polyps reduces the response to oral corticosteroid therapy. J Allergy Clin Immunol. 2012;129(6):1522-8.e5

17. Wallwork B, Coman W, Feron F, MackaySim A, Cervin A. Clarithromycin and prednisolone inhibit cytokine production in chronic rhinosinusitis. Laryngoscope. 2002;112(10):1827-30

18. Bleier BS, Mulligan RM, Schlosser RJ. Primary human sinonasal epithelial cell culture model for topical drug delivery in patients with chronic rhinosinusitis with nasal polyposis. J Pharm Pharmacol. 2012;64(3):44956

19. Tuncer U, Soylu L, Aydogan B, Karakus F, Akcali $C$. The effectiveness of steroid treatment in nasal polyposis. Auris Nasus Larynx. 2003;30(3):263-8.

20. Vaidyanathan S, Barnes M, Williamson $P_{\text {, }}$ Hopkinson P, Donnan PT, Lipworth B. Treatment of chronic rhinosinusitis with nasal polyposis with oral steroids followed by topical steroids: a randomized trial. Ann Intern Med. 2011;154(5):293-302.

21. Shintani Y, Maruoka S, Gon Y, Koyama D, Yoshida A, Kozu Y, et al. Nuclear factor erythroid 2-related factor 2 (Nrf2) regulates airway epithelial barrier integrity. Allergol Int. 2015;64 Suppl:S54-63.

22. Soyka MB, Holzmann D, Basinski TM, Wawrzyniak M, Bannert C, Burgler
S, et al. The Induction of IL-33 in the Sinus Epithelium and Its Influence on T-Helper Cell Responses. PLoS One. 2015:10(5):e0123163.

23. Liu Y, Shao Z, Shangguan G, Bie Q, Zhang B. Biological Properties and the Role of IL-25 in Disease Pathogenesis. J Immunol Res. 2018;2018:6519465.

24. Dogan $M$, Sahin $M$, Yenisey $C$. Increased TSLP, IL-33, IL-25, IL-19, IL 21 and amphiregulin (AREG) levels in chronic rhinosinusitis with nasal polyp. Eur Arch Otorhinolaryngol. 2019;276(6):1685-91.

25. Roan F, Obata-Ninomiya K, Ziegler SF. Epithelial cell-derived cytokines: more than just signaling the alarm. J Clin Invest. 2019;129(4):1441-51.

26. Kamekura R, Kojima T, Koizumi J, Ogasawara N, Kurose M, Go M, et al. Thymic stromal lymphopoietin enhances tight-junction barrier function of human nasal epithelial cells. Cell Tissue Res. 2009;338(2):283-93.

27. Steelant B, Wawrzyniak P, Martens K, Jonckheere AC, Pugin B, Schrijvers R, et al. Blocking histone deacetylase activity as a novel target for epithelial barrier defects in patients with allergic rhinitis. J Allergy Clin Immunol. 2019 Nov;144(5):1242-1253.e7.

28. Wang J, Wen L, Wang Y, Chen F. Therapeutic Effect of Histone Deacetylase Inhibitor, Sodium Butyrate, on Allergic Rhinitis In Vivo. DNA Cell Biol. 2016;35(4):203-8.

29. Martens K, Hellings PW, Steelant B. Calu-3 epithelial cells exhibit different immune and epithelial barrier responses from freshly isolated primary nasal epithelial cells in vitro. Clin Transl Allergy. 2018;8:40.

Michael B. Soyka

University Hospital of Zurich

Department of Otorhinolaryngology

Frauenklinikstrasse 24

8091 Zurich

Switzerland

E-mail: michael.soyka@usz.ch

ISSN: 2589-5613 / @2020 The Author(s). This work is licensed under a Creative Commons Attribution 4.0 International License. The images or other third party material in this article are included in the article's Creative Commons license, unless indicated otherwise in the credit line; if the material is not included under the Creative Commons license, users will need to obtain permission from the license holder to reproduce the material. To view a copy of this license, visit http://creativecommons.org/licenses/by/4.0/ 